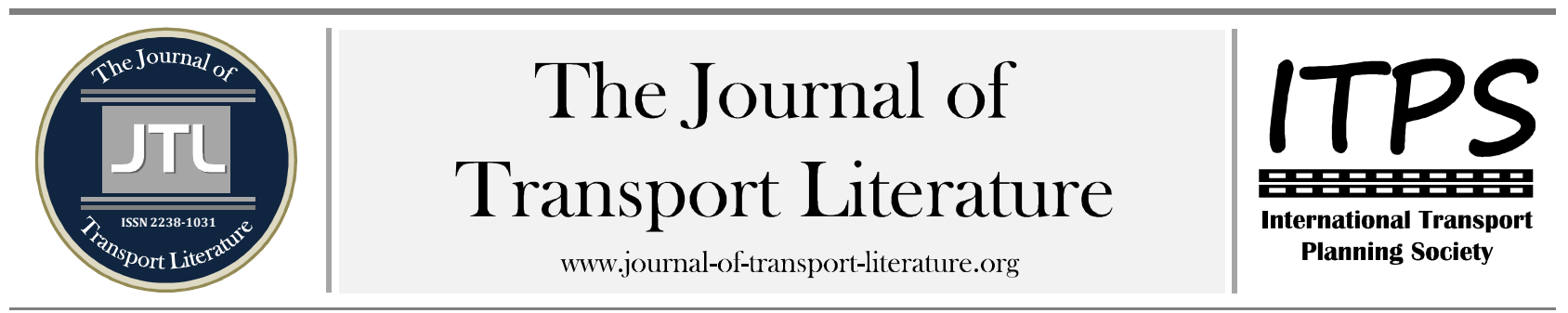

\title{
Recent trends in intelligent transportation systems: a review
}

Bhupendra Singh ${ }^{1}$; Ankit Gupta ${ }^{2,+}$

${ }^{1}$ IIT Roorkee, Roorkee, Uttarakhand, India

2 IIT, BHU, Varanasi, India

\section{Article Info}

Keywords:

Intelligent Transportation

System

Advanced Traveller

Information System

Advanced Traffic

Management System

Advanced Public

Transportation System

Submitted 5 Mar 2014;

received in revised form 10 Sep 2014

accepted 8 Oct 2014.

Licensed under

Creative Commons

CC-BY 3.0 BR.

\section{Abstract}

Managing the increasing traffic is a big problem all over the world. Intelligent Transportation System (ITS) provides solution to these problems with the help of new technologies. ITS is an integrated system that implements a broad range of communication, control, vehicle sensing and electronics technologies to solve and manage the traffic problems. ITS is being used in the developed countries since past two decades, but it is still a new concept when developing countries like India, Brazil, China, South Africa etc. are concerned. In the present study we have studied four major parts of the ITS i.e., Advanced Traveler Information System (ATIS), Advanced Traffic Management System (ATMS), Advanced Public Transportation System (APTS), and Emergency Management System (EMS). Objective of the paper is to study various ITS architecture and model and review such models to get in-depth of their architecture. Hence architecture and developed models over the years of four major branches of ITS have been reviewed here to make a comparison analysis of different models that have been developed by the researchers in their studies. It will lead to the gaps in the knowledge which can be further studied. The paper highlights the conclusions extracted from the studies of different systems and also gives the future scope in the field of ITS to make it more user friendly and accessible.

+ Corresponding author. Civil Engineering Department. IIT (BHU) Varanasi (UP) - 221005, India.

E-mail address: ankit@nith.ac.in.

\section{Introduction}

In the present paper different ITS architecture and model developed by various researchers all over the world has been studied and reviewed to get in-depth of their architecture. In the study the ITS has been sub-divided in four main branches based of their implementation in different aspects of transportation management i.e. ATIS, APTMS, ATMS and EMC. A comparison analysis of the different models developed in all four branches have been done to highlight the plus and minus points of these developed systems and based on that the gaps in the literature have been highlighted.

Last two decades have seen a lot of development in the field of transportation infrastructure even then various traffic problems are increasing day by day. This is mainly due to the increase in number of vehicles. Almost every country of the world whether developing or developed (The classification of developed and developing countries is based on International Monetary Fund's World Economic Outlook Report, April 2012), facing problems in the management of transportation facilities (Singh and Gupta, 2013). The focus of the countries all over the world is shifting from infrastructure development to the best use of the infrastructure facilities available (singh et al., 2014). For the optimum use of the available transportation infrastructure, ITS is being developed and used all over the world.

ITS is an integrated system that implements a broad range of communication, control, vehicle sensing and electronics technologies to help in monitoring and managing traffic flow, reducing congestion, providing optimum routes to travelers, enhancing productivity of the system, and saving lives, time and money. A Technical Note of the World Bank named "ITS for Developing Countries" addresses the condition of ITS in developing countries, and discusses the long-term, society-wide benefits that ITS can provide and ways that ITS can provide more immediate benefits to individuals by helping to make surface transportation more affordable, more reliable, and more efficient (Yokota, T., 2004). It states that ITS proves to be useful in the following manner:

- Improved mobility for people and freight, including greater access to transportation for the elderly, the disabled, and people living in remote locations

- Greater compatibility of surface transportation with the environment

- Fewer traffic-related deaths and injuries

- A better-managed transportation system.

- Less travel uncertainty, allowing for better planned, quicker, and less expensive travel

- So from the above points we can see that ITS covers and improves almost all the aspects of Transportation engineering. There are many subsidiaries of the Intelligent Transportation System out of which most important and widely used all over the world to solve the traffic and transportation problem are as follows: 
- Advanced Traveler Information System (ATIS)

- Advanced Traffic Management System (ATMS)

- Advanced Public Transportation System (APTS), and

- Emergency Management System (EMS)

Advanced Traveler Information System (ATIS) implements a wide range of technologies, such as internet, telephones, cellular phones, television, radio, etc. to assist travelers and drivers in making informed decisions regarding trip departures, optimum routes, and available modes of travel. ATIS provides the drivers both en route and pre-trip information which is advantageous in many ways. Pre-trip information availability enhances the self-belief of the drivers to use freeways and allows commuters to make better-informed transit choices (Campbell et al., 2003). En route information and guidance saves travel time, helps a traveler avoid congestion, can improve traffic network performance. In 1999 a survey was conducted among the people who were using the Advanced Regional Traffic Interactive Management and Information System (ARTIMIS) telephone traveler information service in Cincinnati, Ohio. All of them rated the service as beneficial service. More than $99 \%$ of people surveyed in that city said that they were benefited by avoiding traffic problems, saving time, reducing frustration, and arriving at destinations on time and $81 \%$ said that they had recommended the service to someone else.

Advanced Traffic Management System (ATMS) is used by traffic police department and traffic regulation authorities as a tool to manage and control traffic by monitoring the flow of traffic and making appropriate decisions in a timely manner. Traffic management systems optimize the movement of vehicles, by using real-time information to intervene and adjust controls such as traffic signals to improve traffic flow.

Advanced Public Transportation System (APTS) is concerned with increasing operational efficiency of all public transportation modes and increasing ridership by making the transportation system more reliable. With the help of APTS the way public transportation systems operate is transformed, and the nature of the transportation services that can be offered by public transportation systems is changed.

Emergency Management System (EMS) is the newest research field in intelligent transportation system. EMS is mainly concerned with the application of different intelligent transportation system technologies to develop a transport system which can provide help in the emergency conditions.EMS can provide great help in reducing the fatality rate in the accidents.

With the help of ATMS the congested traffic situations at the roads can be managed, APTMS can help to improve the current situation of public transport by rescheduling and proper management of available fleets to encourage the commuters to use public transport more as compared to private cars and bikes. ATIS can give the users pre trip and enroute information so that they can plan their trip properly, it will help in reducing the time spent by the riders on the roads and also help them to avoid traffic jams on the roads. So in this way A properly designed ITS can help in solving traffic problems. The present paper aims at highlighting the plus and minus of different ITS developed all over the world by comparing their architecture and also highlights the gaps in the literature.

\section{Advanced Traveler Information System (ATIS)}

ATIS is an area of research since past 2-3 decades worldwide. In the 1990's most of the work in this field is mainly done in the U.S.A. and European countries. But since the 21st century, work has been started in the Asian and African counties also. In this section of the literature review studies conducted in India and studies carried out outside India has been reviewed and compared to give advantages and disadvantages of the systems:

Peng(1997) presented a method for designing a Geographic Information Systems (GIS) based Automatic Transit Traveler Information System (ATTIS). The idea behind the study was to provide the users optimal trip option with least travel time between the traveler's origin and destination, including walking, waiting, transfer, and in-vehicle time. To achieve the purpose of providing the optimum route the methodology which was adopted is to consider only those bus stop points which are active (have service) at the time of travel as all the bus stop points don't have the service all $24 \times 7$ and considering only active bus stop point results in optimum route.

Kumar et al. (2005) developed a GIS based advanced traveler information system for the Hyderabad city, India under ArcView GIS environment. GIS-enabled modules for the shortest path, closest facility, and city bus routes were included in the system. The developed system provides information about basic facilities in Hyderabad City.

Hasnat et al. (2006) developed a similar system using web and wireless communication technologies. The system works in two different modules:

- Web based service, which provides the service to the user both in text and map format

- SMS based service, which receives the queries from the users and then provide the user information

In the computation of the total travel time each road/edge is given a weight based on some constraints as traffic jam etc. and on the basis of this the travel time was calculated.

Zhang et al. (2011) in their study developed and tested a generic multimodal transport network model for ATIS applications. First, a multimodal transport networks was modeled from an abstract point of view and networks were categorized into private and public modes then a generic method was used to construct a multimodal transport network representation by using transfer links which was inspired by the super-network technique. The biggest problem with the developed system was the time taken for the route determination by integration of different modes.

\subsection{Discussion}

GIS is a powerful platform for the spatial analysis of the data. Hence, the ATIS which were developed on the GIS platform by Peng (1997) and Kumar et al. (2005), provided a much better spatial analysis on the other hand World Wide Web (WWW) is very useful in providing the real time information about the different incidents, so WWW based ATIS by Hasnat et al. (2006), Wu et al. (2003) and Singh and Kumar (2010) were able to provide real time information. Pal and Singh (2011) developed the ATIS which was based on the integration of both GIS and WWW technology so it was able to provide the benefits of both the technologies. Peng (1997) in his study incorporated the idea of providing the optimal trip to the user 
with respect to time while no such parameter was considered by Kumar et al. (2005). They considered distance as the only parameter for finding out the optimal path. ATIS developed by Kumar et al. (2005) didn't provide any real time information but it was able to provide information about the different facilities of the city whereas other ATIS were able to provide the information regarding the transportation facilities only. One big advantage that ATIS developed by Wu et al. (2003) holds on the other system that it also incorporates a lost data construction technique. ATIS developed by Wu et al. (2003) and Hasnat et al. (2006) can be accessed by the simple mobile phone whereas other ATIS can only be accessed by the personal computers which restricts the use of ATIS in developing countries because of the non-availability of personal computers to every person. Zhang et al. (2011) provides the optimum routes by integration of the different modes but it also resulted in more time taking in the determination of shortest route, whereas other ATIS considered only one mode of transportation to give the required information.

\section{Advanced Traffic Management System (ATMS)}

ATMS is one of the most prominent and used branch of ITS. This section of the literature review includes the different studies which have been made in the field of ATMS to give the comparative discussion of the studies:

Logi and Ritchie (2001) described a real-time Knowledge Based System (KBS) for decision support in the selection of integrated traffic control plans after the occurrence of non-recurring congestion. In this study, two algorithms were developed i.e. data fusion algorithm for the analysis of congestion and an algorithm for the selection of control plans. The validation results showed that by the use of Traffic Congestion Management (TCM) travel time reduced between $1.9 \%$ and $29.0 \%$ and average stop speed reduced between $14.8 \%$ and $55.9 \%$.

Faghri and Hamad (2002) studied the use of GPS in traffic management. In their study application of GPS was involved in collecting traffic data such as travel time, speed and delay on 64 major roads in the state of Delaware. When mean and variance of the results obtained by both the methods were compared and no significant difference was observed. GPS data was found to be $50 \%$ more efficient in terms of manpower.

Hernandez et al. (2002) incorporated the use of artificial intelligence techniques in traffic management and gave a multiagent architecture for intelligent traffic management systems. Two multi-agent knowledge based systems, InTRYS and TRYSA2 were developed to perform decision support for real-time traffic management. The performance of both the systems was evaluated and general applicability of multi-agent architectures for intelligent traffic management was given.

Zhenlin et al. (2012) studied the efficiency of the Beijing Intelligent Traffic Management System (ITMS). In this study urban transportation systems, socio-economic system and energy environment system were taken as the input system and the road traffic management efficiency and urban transport putting indicators as the output system. The field data of Beijing from 2000 to 2010 are used for empirical analysis. The results of the study showed that the ITS improved the overall efficiency of the Beijing transportation.

\subsection{Discussion}

Hernandez et al. (2002) incorporated the use of artificial intelligence whereas Balaji and Srinivasan (2011) used type-2 fuzzy decision module for traffic management operations which provides more autonomy to the system and less need of manpower. Mulay et al. (2013) gave the traffic management system which provides facility of congestion detection and management, IPTS and signal synchronization whereas ATMS developed by Balaji and Srinivasan (2011) provides only the traffic signal control for the management of traffic. Logi and Ritchie (2001) and Ossowski et al. (2005) gave decision support system (DSS) for traffic management. The former was based on the knowledge based system while the later was based on multi-agent technology. Logi and Ritchie (2001) used Traffic Congestion Management (TCM) approach which estimates current traffic conditions using the result of a static assignment based on historical O-D data that represent daily traffic pattern under different conditions. This is a fast but inaccurate assessment of current demand. Adoption of dynamic approach assignment will be better approach for calculating current demand. System developed by Logi and Ritchie (2001), Hernandez et al. (2002), Ossowski et al. (2005) and Mulay et al. (2013) were capable of handling different traffic incidents through different methods, but the system developed by Balaji and Srinivasan (2011) was traffic signal control system to optimize the signal traffic to reduce congestion. It was unable to handle any traffic incidents. Zhenlin et al. (2012) studied the efficiency of the Beijing Intelligent Traffic Management System (ITMS). They found that effectiveness of transportation facilities have considerably increased after the implementation of ATMS. The study by Faghri and Hamad (2002) was more of a basic study, as they did not developed any system but only studied the use of GPS in traffic management and found out that GPS data to be $50 \%$ more efficient in terms of manpower. This proves the importance of GPS technology in traffic management operations.

\section{Advanced Public Transport Management System (APTMS)}

Present section consists of the critical review of the studies that have been made in the field of APTMS to give critical analysis of different systems:

Molina (2005) described the architecture of a computer system that followed the idea of a knowledge based intelligent assistant for public transport management. The given architecture serves three purposes i.e. diagnosis, prediction and planning. The model described, was applied for the development of two different real-world applications for the cities of Torino (Italy) and Vitoria (Spain).

Feizhou et al. (2008) used Genetic Algorithm (GA) and Hybrid Genetic Algorithm (HGA) for optimal scheduling of public vehicles based on the actual operational environments. The performance for three kinds of vehicular levels (i.e. minimum, average and maximum) was compared using one-point and two-point crossover operations. Bus route 375 in Beijing was taken by the researchers for example and the system was applied. The results showed that it helped to reasonably allocate public vehicle resources and improved efficiency.

Hatem et al. (2009) proposed a system which integrated Radio Frequency Identification (RFID) in Wireless Sensor Network (WSN) to manage the public transportation. The system proposed to equip the entrance and exit with RFID reader antennas and monitor sensors and each bus with an Ultra High Frequency (UHF) tag. So whenever a bus enters or exits the 
bus stations RFID reader sends its identification to central computer and then this information is displayed on the LED boards for the users and can be used to schedule and manage the bus service.

He and Zhang (2009) introduced a public transport dispatch and decision support system based on multi-agent. The proposed system uses personal computers as user interface to take user's queries and provide answers.

\subsection{Discussion}

The system developed by Feizhou et al. (2008) provides scheduling of public vehicles and it didn't cover the other aspects of the public transport management which restricts its use as PTMS. . Hatem et al. (2009) and Zhian and Han (2010) developed a bus management system based on the integration on new emerging technologies. Hatem et al. (2009) proposed the use of integrated RFID (Radio Frequency Identification) in WSN (Wireless Sensor Network) whereas Zhian and Han (2010) proposed the use of Zigbee and GSM/GPRS technologies. Zigbee technology is economical as compared to the RFID (Radio Frequency Identification) but it has a range only of 10 meter which was a big drawback. Both the systems only give the position of the bus when it is near to the bus stops so if any incident happens between the bus stops it won't be able to detect the problem; this restricts their use as an effective PTMS. System developed by Ramesh et al. (2012) was also for bus scheduling and dispatching system, but this was based on the number of passengers available on the each bus stop. This system is only advantageous for the stops which are closer to bus terminals otherwise the dispatched bus will take much time and by this time the number of passengers at the stop will increase. System developed by Molina (2005) was more of a complete system covering many aspects of public transport management i.e. diagnosis, prediction and planning whereas the other systems only concentrated only any one aspect of traffic management i.e. scheduling of buses, management of bus routes etc.

\section{Emergency Management System (EMS)}

Due to its nature and contribution in saving lives and money a lot of work is happening in the field of EMS. This section discusses the different EMS developed all over the world to give critical review:

Thapar (2001) presented a GIS based emergency response management system for Hyderabad city which can provide the useful information regarding different facilities and optimum routes during emergency situations. In this study the probable risk zones were determined based on the land use, building activities as per National Building Code (NBC) guidelines. Efficiency and effectiveness of the fire service was studied and based on this an Emergency Response Management System was developed.

Kejun et al. (2008) gave the architecture of a GIS based Emergency Accident Rescue System (EARS) in freeways. Two models were developed and used in the system:

- First was early warning and positioning the traffic accident.

- Second was to automatically generate rescue plan.

Ganeshkumar and Ramesh (2010) designed Emergency Response Management and Information System (ERMIS) for Madurai city, Tamil Nadu. In this study a detailed GIS database of transportation network, accident locations, hospitals, ambulance locations, police and fire stations was prepared and spatial analysis was also carried out for accident records of years 2004-2008. Route finder was designed to find shortest, time saving routes and service areas.

Purushothaman et al. (2011) proposed a similar GIS based Emergency Response Management System for Mysore City, India. The developed system provides the network based spatial analysis such as connectivity, finding paths, allocation, finding the closest facility, defining service areas, dynamic segmentation.

\subsection{Discussion}

Thapar (2001), Kejun et al. (2008), Wang et al. (2008), Ganeshkumar and Ramesh (2010), Purushothaman et al. (2011), Ramachandran and Devi (2011) gave EMS based on the GIS platform. GIS platform provides very good spatial analysis power which makes it favorite for development of EMS. Thapar (2001) took Hyderabad city, Ganeshkumar and Ramesh (2010) took Madurai city in Tamil Nadu, Purushothaman et al. (2011) took Mysore city as their study areas and used GIS as the platform for the software development. None of the system was able to provide real time information about the facilities which is very essential in emergency situations. Fire emergency being the most common in the developing countries, Thapar (2001) concentrated his study on fire accidents while Kejun et al. (2008), Wang et al. (2008), and Deqi et al. (2011) concentrated on traffic accidents. Ganeshkumar and Ramesh (2010), Purushothaman et al. (2011) and Ramachandran and Devi (2011) included the information about the basic facilities such as roads, police stations, hospitals, fire stations etc. but in the system developed by Ramachandran and Devi (2011) additional information about the accidents happened in the past has also been included.

\section{Conclusion}

From the above discussion it is clear that most of the ITS studies have been based in the developed countries. In developing countries still much work is needed in this field to solve the emerging traffic related problems. Most of the ITS systems have been developed on the GIS or WWW platforms. Both the platforms have their own advantages. While GIS platform provides very powerful spatial analysis techniques whereas WWW platform prove to be very useful in providing real time information. Some developed systems used integration of both GIS as well as WWW platforms which makes it possible to use the advantages of both the platforms. Most of the developed systems are based in the urban areas. There is a great scope of work to be done in rural areas. The GPS is very useful in all the ITS systems. GPS data is $50 \%$ more efficient in terms of manpower. It was found in the literature that with the use of ATMS technology the travel time was reduced around $1.9 \%-29.0 \%$ and average stop speed reduced around $14.8 \%-55.9 \%$. With the use of EMS the responding speed applied to system is increased around $50 \%$ and the total time needed to handle the emergency event was decreased around $40 \%$ than the other traditional method.

In the development of ITS, integration of the different modes of transportation is very necessary. Hence, the work should be done in this field. In ITS real time information is a very important factor. GPS is one technology which can help in this direction so the work needed to be done towards making GPS more accurate and economical. In all the developing countries 
mixed traffic conditions prevails, so in the development of the ITS mixed traffic conditions should also be considered in order to make it applicable in developing countries also. In implementation of the ITS especially in developing countries its installation and operating cost are very big factors. Hence, the work should be done in the direction to make the ITS more economical.New emerging technologies like Zigbee and RFID can be helpful in this direction. The reach of ITS till now is limited to few persons so the work should be done in order to make it more accessible and user friendly. The use of mobile phones can be really helpful in this direction as mobile phones are most commonly used electronic gadget all over the world.

\section{References}

Balaji, P. G., \& Srinivasan D. (2011). Type-2 Fuzzy Logic Based Urban Traffic Management. Engineering Applications of Artificial Intelligence, 24, 12-22.

Campbell, J. L., Carney, C., \& Kantowitz, B. H. (2003). Human Factors Design Guidelines for Advanced Traveler Information Systems (ATIS) and Commercial Vehicle Operation (CVO). Federal Highway Admin., McLean, VA, Rep. FHWA-RD-98-057-2.

Deqi, H., Xiumin, C., \& Zhe, M., (2012) A Simulation Framework for Emergency Response of Highway Traffic Accident. Procedia Engineering $29,1075-1080$.

Faghri, A., \& Hamad, K., (2002). Applicition of GPS in Traffic Management Systems, GPS Solutions, 5(3), 52-60.

Feizhou, Z., Xuejun, C., \& Dongkai, Y., (2008) Intelligent Scheduling of Public Traffic Vehicles Based on a Hybrid Genetic Algorithm. Tsinghua Science and Technology, ISSN 1007-0214 09/25, 13(5), 625-631.

Ganeshkumar, B., \& Ramesh, D., (2010). Emergency Response Management and Information System (ERMIS) - A GIS Based Software to Resolve the Emergency Recovery Challenges in Madurai City, Tamil Nadu. Int. Journal of Geomatics and Geosciences, 1(1), 1-13.

Growth Resuming, Dangers Remain, (2012). World Economic Outlook, Report of International Monetary Fund (IMF), United Nations, Washington, DC, USA.

Hasnat, M. A., Haque, M. M., \& Khan M. (2006). GIS Based Real Time Traveler Information System: An Efficient Approach to Minimize Travel Time Using Available Media, available from www.bracu.ac.bd.

Hatem, B. A., \& Habib, H. (2009). Bus Management System Using RFID In WSN in the proceedings of European and Mediterranean Conference on Information Systems (EMCIS), April 12-13 2009, Abu Dhabi, UAE.

He, Z., \& Zhang, Q. (2009). Public Transport Dispatch and Decision Support System Based on Multi-Agent. In the proceedings of Second International Conference on Intelligent Computation Technology and Automation, Zhangjiajie, China.

Hernandez, J.Z., Ossowski, S., \& Garcýa-Serrano A. (2002). Multiagent Architectures for Intelligent Traffic Management Systems, Transportation Research Part C, 10, 473-506.

ITS for Developing Countries. Accessed from siteresources.worldbank.org.

Kejun, L., Yong, L., \& Xiangwu, L., (2008). Emergency Accident Rescue System in Freeway Based on GIS, in the proceedings of International Conference on Intelligent Computation Technology and Automation.

Kumar, P., Singh, V., \& Reddy, D., (1999). Advanced Traveler Information System for Hyderabad City. IEEE Transactions on Intelligent Transportation Systems, 6(1), 26-37.

Logi, F., \& Ritchie, S. G. (2001). Development and Evaluation of a Knowledge-Based System for Traffic Congestion Management and Control. Transportation Research Part C, 9, 433-459.

Thapar, M. S. (1999). Emergency Response Management System for Hyderabad city. Accessed from www.gisdevelopment.net.

Molina, M. (2005). An Intelligent Assistant for Public Transport Management. Proc. International Conference on Intelligent Computing (ICIC 2005), Part II, LNCS 3645, 199-208.

Mulay, S. A., Dhekne, C. S., Bapat, R. M., Budukh, T. U., \& Gadgil, S. D. (2013). Intelligent City Traffic Management and Public Transportation System. International Journal of Computer Science Issues, 10(3), 46-50.

Ossowski, S., Hernandez, J. Z., Belmonte, M. V., Fernandez, A., Garcia-Serrano, A., Perez-de-la-Cruz, J., Serrano, J. M., \& Triguero, F. (2005). Decision Support for Traffic Management Based on Organisational and Communicative Multiagent Abstractions. Transportation Research Part C, 13, 272-298.

Pal, S. \& Singh, V. (2011). GIS Based Transit Information System for Metropolitan Cities in India. In the proceedings of Geospatial World Forum, 18 - 21 January 2011, Paper Reference No.: PN-250.

Peng, Z. R. (1997). A Methodology for Design of a Gis-Based Automatic Transit Traveler Information System. Comput., Environ. and Urban Systems, 21(5), 359-372.

Purushothaman, B. M., Arunachalam, S., Srinivasan, R., \& Suresh Babu, S. (2011). Emergency Response Management System for Mysore City. International Journal of Earth Sciences and Engineering, 4(1), 48-54.

Ramachandran, N., \& Devi, G. (2011). Accident Emergency Response and Routing Software (AERARS) Using Genetic Algorithm. International Journal on Computer Science and Engineering (IJCSE), 3(7), 2835-2845.

Ramesh, R., Ezhilarasu, Y., Ravichandran, P., \& Prathibha, S. (2012). Regulating Bus Management System Using Cloud Platform. International Journal of e-Education, e-Business, e-Management and e-Learning, 2(6), 517-520.

Shumin, S, Zhaosheng, Y., \& Maolei, Z. (2010). A Decision Support System of Urban Traffic Emergency Control Based on Expert System. in the proceedings of IEEE Int. Conference on Software Engineering and Service Sciences (ICSESS), 16-18 July 2010, Beijing, 221 - 225.

Singh, B. \& Gupta, A. (2013). Advancements in Intelligent Transportation Systems: A Review. Proceedings of International Conference on Advanced Trends in Engineering and Technology (ICATET), Jaipur, 375-383

Singh, B., Gupta, A. \& Suman, S. (2014). Framework for Development of Advanced Traveler Information System: A Case Study for Chandigarh City. Proceedings of International Conference on Transportation Planning and implementation Methodologies for Developing Countries 2014 (TPMDC-2014), Submission Id. 54.

Singh, V. \& Kumar, P. (2011). Web-Based Advanced Traveler Information System for Developing Countries. Journal of Transportation Engineering, 136(9), 836-845.

Wang, G., Xiao, D., \& Gu, J. (2008). Design and Implementation on Software Platform of Emergency Management System for Traffic Incidents, in the proceedings of CCECE/CCGEI May 5-7 2008, Niagara Falls, Canada.

Wu, C. H., Su, D. C., Chang, J., Wei C. C., Ho, J. M., Lin, K. J., \& Lee, D.T. (2003). An Advanced Traveler Information System With Emerging Network Technologies. In the proceedings of 6th Asia-Pacific Conf. Intelligent Transportation Systems Forum.

Zhang, J., Liaoa, F., Arentzea, T., \& Timmermansaa H. (2011). A Multimodal Transport Network Model for Advanced Traveler Information Systems. Procedia Computer Science, 5, 912-919.

Zhenlin, W., Peng, Z., \& Shulin, A. (2012). Efficiency Evaluation of Beijing Intelligent Traffic Management System Based on super-DEA. Journal of Transportation Systems Engineering and Information Technology, 12(3).

Zhian, L., \& Han, H. (2010). A Bus Management System Based on ZigBee and GSM/GPRS. in the proceedings of International Conference on Computer Application and System Modeling (ICCASM 2010), Taiyuan, China. 